\title{
Designs of Double whip Antenna for Mobile Phone
}

\author{
P. Prakash, K.Parthiban, K.Sasikumar \\ ECE Department V.S.B Engineering College, Karur \\ ECE Department V.S.B Engineering College, Karur \\ ECE Department V.S.B Engineering College, Karur
}

\begin{abstract}
Whip type antennas are probably the most commonly used antennas in portable RF systems, such as cordless and cellular phones, $R F$ enabled laptop computers, , and handheld computers. Whip antennas are almost always mounted on the chassis which contains the radio and other electronics. Normally in mobile phone two set of antenna used for receiving different application such as GSM and GPS. In this paper models and measurements are used to discuss choice of using single Double whip antennas and the impact of the chassis on radiation characteristics. Ansoft HFSS tool which has been has been successfully used to predict radiated field patterns is used for simulations, and measured and modeled results are shown.
\end{abstract}

Index Terms: Double whip antenna, VSWR, S-Parameters, Ansoft HFSS Tool.

\section{INTRODUCTION}

Communication can be broadly defined as the transfer of information from one point to another .A communication system is usually required when the information is to be conveyed over a distance. The transfer of information within the communication system is commonly achieved by superimposing or modulating the information onto an electromagnetic wave which act as a carrier for the information signal. At the required destination, the modulated carrier is then received and the original information signal can be recovered by demodulation. Over the years, sophisticated techniques have been developed for this process using electromagnetic carrier waves operating at radio frequencies as well as microwave and millimeter wave frequencies

In today's modern communication industry, antennas are the most important components required to create a communication link. Through the years, microstrip antenna structures are the most common option used to realize millimeter wave monolithic integrated circuits for microwave, radar and communication purposes[1].Due to its many advantages over the conventional antenna. The microstrip antenna have achieved importance and generated interest to antenna designers for many years. In fact, active microstrip antenna arrays and active apertures are increasingly present in phased array radar applications. In addition, these devices also serve as potentially efficient power combiners. Hence, active microstrip antennas arrays are often used in spatial or "quasi-optical" [2] combining schemes for creating high power and high-frequency components. Furthermore, microstrip antennas are often used in military air craft, missile, rocket and satellites.

Zhang, Sun, Li, Wang[3] and Xue worked on the design of an antenna with a stable Stellenbosch University http://scholar.sun.ac.zaimpedance and radiation pattern over a wide frequency range. Methods to reduce the dimensions of antennas were also investigated and a monopole antenna with a

Length less than $\lambda / 4$ was introduced in their pape[4]r. The antenna becomes capacitive at low frequencies; it requires the use of a compensatory inductor to obtain real input impedance. The term adopted where the impedance has only a small imaginary part is "resonance".

The poor impedance matching of the antenna"s input impedance due to the small radiation resistance at the lower frequencies will also hinder the antenna "s abilities to radiate energy due to the high reflection coefficient. By adding inductive series elements and changing the position and values of the lumped elements the antennas current distribution and effective radiation pattern amplitude is changed, allowing wideband behavior to be obtained. The monopole antenna $\mathrm{s}$ bandwidth was extended and the dimension was reduced when it was loaded with several lumped elements composed of resistors, inductors radiator. The antenna"s efficiency was however low over the whole frequency range as its $\mathrm{Q}$ factor could not break through the Chu-Harrington limit where the Q value of a small antenna is relatively equal to the volume of a sphere that encloses it, thus the antennas band width will shrink. They used a technique presented by [5]Sussman-Fort and Rudish[6] regarding non-Foster impedance matching. Networks of negative inductors and capacitors were used to produce a non-Foster matching network where the network was used to bypass the restrictions of gain-bandwidth theory. The concept adopted here is the addition of a variable inductor (non-Foster circuit) to try extending the "resonant" behavior. The nonFoster circuit when compared to passive matching achieved higher efficiency over a wider bandwidth. The antenna"s current distribution and radiation pattern stays unaffected by the use of a non-Foster circuit. 


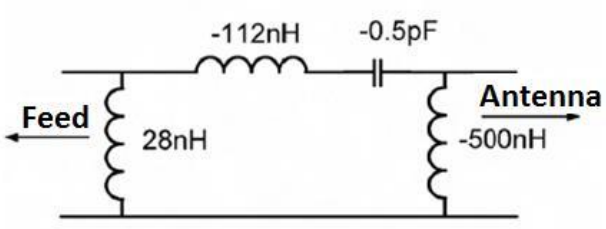

Fig1: non-Foster circuit schematic

\section{MiCROSTRIP ANTENNA}

In today's aircraft and spacecraft application where the antenna's size, weight, cost, performance, ease of installation and aerodynamics profile are of utmost consideration low-profile microstip antenna is preferred over conventional antennas [7]. The term 'microstrip' actually refers to any type of open wave guiding structure which is not only a transmission line but also used together with other circuit components like filter ,couplers, resonators, etc .In fact, microstrip antennas are an extension of the microstrip transmission line. Microstrip antennas can be flush-mounted to metal or other existing surfaces, and they only require space for the feed line, which is usually placed behind the ground plane. On one side of dielectric substrate, this has a ground plane on the other side. The patch conductors usually made of copper or gold can b virtually assumed to be of any shape. However, conventional shapes are normally used to simplify analysis and performance prediction. The radiating elements and the feed lines are usually photo etched on the dielectric substrate

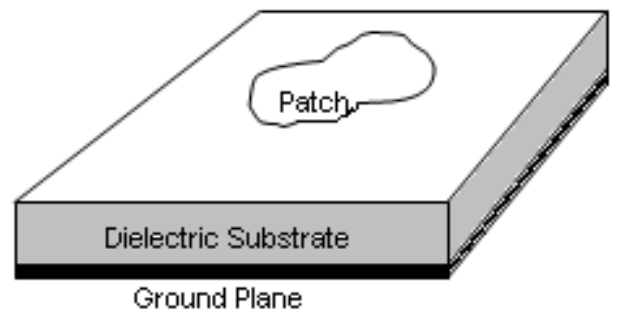

Fig 2. Microstrip Patch Antenna

The upper surface of the dielectric substrate supports the printed conducting strip while the conducting ground plane backs the printed conducting strip while the conducting ground plane backed the entire lower surface of the substrate. The radiating patch may be square, rectangular, circular, and elliptical or any other configuration. Square, rectangular and circular shapes are the most common because of ease of analysis and fabrication. As for the feed line, it is also a conducting strip; normally of a smaller width coaxial line feeds where the inner conductor of the coax is attached to the radiating patch are also widely used. Sometimes, microstrips are also referred as printed antennas. For mobile phones two set of antennas are used to cover GSM and GPS, while using micro-strip antenna. It has high return loss and maximum reflection co-efficient. Hence the loss input data will be more in micro-strip antenna.

\section{WHIP ANTENNA}

A whip antenna provides exceptional overall performance and stability, has an isotropic pattern, a wide bandwidth, it is cheap and it is easily designed. Since a full-wave or even a half-wave dipole whip is generally quite long, most whips are $1 / 4$ waves. This simple and most effective small antenna is also called a quarter-wave monopole and is the most common antenna on today's portable devices. Since most devices have a circuit board anyway, using it for half of the antenna can make lot of sense. Generally, this half of the antenna will be connected to ground and the transmitter or receiver will reference it accordingly, Plane with the antenna circuit. Coaxial feeding is employed to provide equal phase and equal amplitude excitation to the three elements of the array

In this antenna we can get more than two operating frequency due to this double bandwidth is possible. Operating frequencies are $(1 \mathrm{GHz}, 3 \mathrm{GHz})$. It can be applied in mobile phone to cover GSM network and GPS. Due to this we can avoid excess antenna used in mobile phone. Normally for $1 \mathrm{GHz}$ frequency half wavelength is 0.468 feet and for $3 \mathrm{GHz}$ frequency 0.156 feet but it now possible in one antenna. Rogger duroid used as a substrate material and it covers $60^{\circ}$ to $170^{\circ}$. 


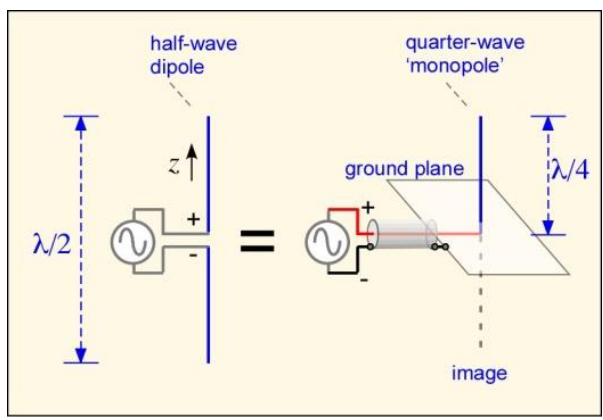

Fig 3. Whip Antenna Model

\section{DESIGN METHODOLOGY}

Before done any simulation, the antenna parameter must be calculate to get the best performance and operating frequency like expect. This stage were very important for the designing any antenna.

In the following theoretic analysis, input impedance,

$$
Z_{\mathrm{inT}-2}=Z_{04} \frac{1+\left|\Gamma_{2}\right| \exp \left\{j\left(\phi_{2}-2 \beta L_{4}\right)\right\}}{1-\left|\Gamma_{2}\right| \exp \left\{j\left(\phi_{2}-2 \beta L_{4}\right)\right\}}
$$

Where $\Gamma_{2}=\left(Z_{2, i n}-Z_{04}\right) /\left(Z_{2, i n}+Z_{04}\right)=\left|\Gamma_{2}\right| \exp \left(j \phi_{2}\right)$

is the reflection co-deficient at the port of the whip with length $L_{2}$.For the purpose of matching, $Z_{\mathrm{inT}}$ must be real and the exponent satisfies the condition $\phi_{2}-2 \beta L_{4}=2 n \pi$

So the solutions to $\beta L_{4}$ is given by

$$
\beta L_{4}=\frac{\phi_{2}}{2}-n \pi
$$

Because the electrical length of the embedded transmission line

$\beta L_{4}$ cannot be negative, and the periodicity of the wit, so one solution to $\beta L_{4}$ is

Because the input impendence $Z_{2}$ varies with frequency,

$$
\beta L_{4}=\pi+\frac{\phi_{2}}{2}
$$

from we can notice that: for any whip antenna, in order to match it with the aid of the embedded transmission line, $\beta L_{4}$ the electrical length of the embedded line should be variable as the working frequency changes. However, as a matter of fact, the length of the embedded line must be fixed in engineering design. In other word, for a given length and characteristic impedance of an embedded transmission line, it can only match the whip antenna in a narrow band. In order to give attention to broadband matching, we can take an average value of $\beta L_{4}$ over the whole matching bandwidth as the length

of the embedded transmission line, so that we can obtain an approximate matching for the element.

\section{Simulation Results}

By using HFSS software, the antenna will be modeled and are simulated. The Double whip antenna are simulated at a frequency range of $1.78 \mathrm{GHZ}$ and2.3GHz has been fabricated with a high dielectric constant substrate material(Rogers TMM 4(tm) with dielectric constant $\varepsilon_{r}=4.7$ ), substrate thickness of $5 \mathrm{~mm}$. The various parameters obtained from these are,

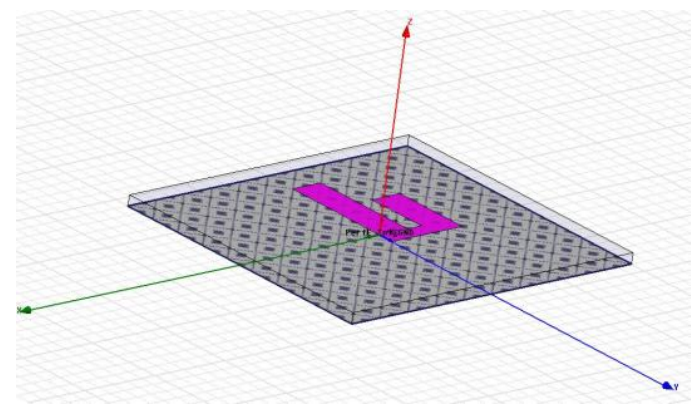

Fig 4. Antenna Model Using HFSS 


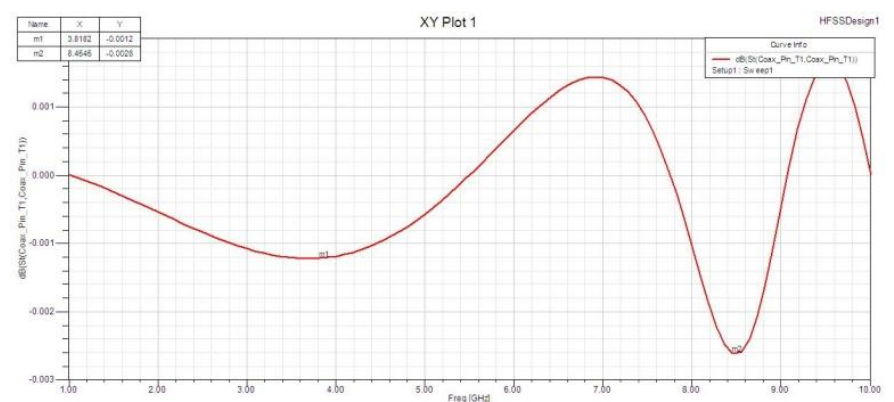

Fig 4. Reflection coefficient of double whip antenna

The reflection coefficient shows that at a frequency of around $1.7 \mathrm{GHz}$ the reflection coefficient reduces to $32.68 \mathrm{~dB}$. and for $2.3 \mathrm{GHz}$ the reflection coefficient reduces to $-40.21 \mathrm{~dB}$
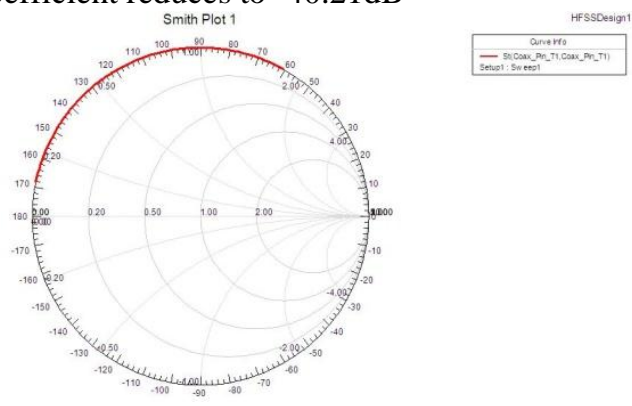

Fig 5. Smith chart for double whip antenna

The smith chart shows that radiation pattern covers $\quad 60^{\circ}$ to $170^{\circ}$

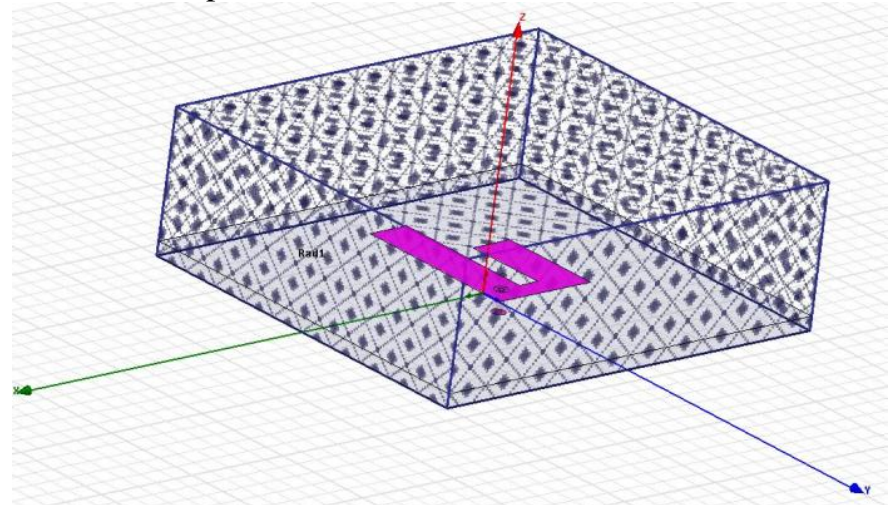

Fig 6. Radiation boundary of double whip Antenna

\section{CONCLUSION}

A Double whip antenna is designed, simulated, fabricated and measured operating at $1.78 \mathrm{GHz}$ and2.3GHz.. The return loss of $-32.68 \mathrm{~dB}$ and $-40.21 \mathrm{~dB}$ and gain of $4.94 \mathrm{~dB}$ is achieved by the proposed antenna. So the designed antenna found the application in receiving GSM, GPS signal in mobile phone and wireless system.

\section{REFERENCES}

[1]. S.D.Rogers,C.M.Butler,andA.Q.Martin,"DesignandrealizationofGA-optimized wire monopole and matching network with 20:1 bandwidth" IEEE Trans. Antennas Propag., vol. 51, no. 3, pp. 493-502,Mar. 2003.

[2]. K. Yegin and A. Q. Martin, "Very broadband loaded monopole antennas," in Proc. IEEE Antennas and Propag. Soc. Int. Symp., Montreal,

[3]. X.Ding,B.Z.Wang,G.Zheng,andX.M.Li,"Designandrealization of a GA-optimized VHF/UHF antenna with 'on-body' matching network,” IEEE Antenna Wireless Propag. Lett., vol. 9, pp. 303-307,2010

[4]. J. M. González-Arbesú, S. Blanch, and J. Romeu, "Are space fling curves efficient small antennas," IEEE Antennas Wireless Propag. Lett., vol. 2, pp. 147-150, 2003.

[5]. H. Lizuka and P. S. Hall, "Left-handed dipole antennas and their implementations," IEEE Trans. Antennas Propag., vol. 55, no. 5, pp.1246-1253, 2007.

[6]. S. Sheldon and W. P. K. Ronold, “Compact conical antenna for wideband coverage,” IEEE Trans. Antennas Propag., vol. 42, no. 3, pp.436-439, 1994.

[7]. L. Reinhold and B. Pavel, "RF Circuit Design: Theory and Applications". Englewood Cliffs, NJ: Prentice Hall, 2000. 